\title{
SISTEMA DE DEPENDENCIA Y APOYO FAMILIAR EN PACIENTES CON TRASTORNO MENTAL GRAVE EN LA PROVINCIA DE CÓRDOBA: UN ENFOQUE DESDE EL TRABAJO SOCIAL SANITARIO
}

\section{THE DEPENDENCY AND FAMILY SUPPORT SYSTEM FOR PATIENTS WITH SEVERE MENTAL DISORDER IN THE PROVINCE OF CÓRDOBA: A SOCIAL HEALTH CARE APPROACH}

José Manuel Jiménez Rodríguez

Equipo de Salud Mental Comunitaria (ESMC) de Palma del Río. Córdoba

\begin{abstract}
Resumen: El trastorno mental grave queda integrado en el conjunto de diagnósticos clínicos del grupo de las psicosis y otros diagnósticos relacionados. Enfermedad cuyo pronóstico lleva aparejado periodos variables de agitación e inestabilidad psíquica respecto de las capacidades funcionales, requiere de un tratamiento integral donde paciente, personal sanitario y familia se ven implicados. Con la reforma psiquiátrica, en España desaparecen los antiguos centros psiquiátricos (manicomios), configurándose una nueva red asistencial de carácter sociosanitario que vendrá a dotar de mayor protagonismo al paciente y su familia. Desde este momento se establece un profundo compromiso social y sanitario hacia la enfermedad, quedando con ello instaurado un nuevo sentir y una nueva conciencia del deber cumplido. La actual crisis económica ha provocado el debilitamiento de los servicios y prestaciones sanitarias y sociales, entre las que se encuentran las ayudas a la dependencia. Consecuencia de ello es el recrudecimiento del apoyo y ayuda de los familiares/cuidadores de estos pacientes que experimentan la sobrecarga y sobre esfuerzo de los cuidados. Se recomienda enfatizar sobre este colectivo el cual puede verse inmerso en situaciones de alto riesgo sociosanitario.
\end{abstract}

Palabras clave: Trastorno Mental Grave (TMG), Cuidados de larga duración, Salud Mental, Dependencia, Trabajo Social.

\begin{abstract}
Severe mental disorder refers to the group of clinical diagnoses consisting of psychotic disorders and related diagnoses. This group of illnesses, the prognosis of which is variable periods of mental agitation and instability with respect to functional capacities, requires comprehensive treatment that involves the patient, health care professionals and the family. When the psychiatric care system in Spain underwent major reform some years ago, the old psychiatric centres (insane asylums) disappeared and a broad network of health and social care services was established. This network gave patients and their families a greater role and showed a profound engagement with the illness, in terms of both social care and health care, and this brought with it a new attitude and feeling of accomplishment. The current economic crisis has led to the weakening of health and social services and of related benefits, including economic assistance for dependent persons.
\end{abstract}


In consequence less support and assistance go to patients' families/caregivers, who thus have greater difficulty meeting the demands of care-giving. This group should be the object of special attention because it is often in situations of high risk.

Key words: Severe Mental Disorder (SMD), Long-term care, Mental health, Dependence, Social Work.

| Recibido 09/10/2014 | Revisado 25/12/2014 | Aceptado 02/02/2015 | Publicado 31/05/2015 |

Correspondencia: José Manuel Jiménez Rodríguez. SAS ESMC de la Palma del Río. Córdoba. Avda. Félix Rodríguez de la Fuente s/n. Correo electrónico: jose.jimenez.rodriguez.sspalma.andalucia.es.

Referencia normalizada: Jiménez-Rodríguez, J.M. (2015). Sistema de dependencia y apoyo familiar en pacientes con trastorno mental grave en la provincia de Córdoba: Un enfoque desde el trabajo social sanitario. Trabajo Social Hoy, 75, 7-16. doi 10.12960/TSH.2015.0007. 


\section{INTRODUCCIÓN}

Hablar del trastorno mental grave (TMG) implica centrar nuestra atención en el conjunto de diagnósticos clínicos del grupo de las psicosis (esquizofrenia y trastorno bipolar) así como de otros diagnósticos relacionados, según la Clasificación Internacional de Enfermedades (CIE-10, citado en la Organización Mundial de la Salud, 2003). Se trata de una psicopatología que altera la consciencia de la persona que la padece y la limita cuando este (el trastorno) se encuentra en estado de activación. El trastorno mental grave queda definido de esta manera como la alteración que contempla las dimensiones: diagnóstico clínico, duración del trastorno (cronicidad) y nivel de discapacidad social, familiar y laboral de la persona afectada (Schinnar et al., 1990: 1602-8; Gisbert et al., 2002; Laviana, 2006: 345-73). Dicha enfermedad hace que se vean afectadas diversas parcelas inherentes al individuo por lo que su tratamiento requiere de una intervención basada en el modelo o paradigma bio-psico-social. Durante los periodos de agitación y hasta llegar a la estabilidad clínica, el paciente se encuentra en situación de vulnerabilidad y dependencia, requiriendo apoyo y supervisión para determinadas actividades de la vida diaria (AVD) (IMSERSO, 2007). Desde que se implantó en España la reforma psiquiátrica en la década de los ochenta, el paciente en sí, como la familia, adquiere un fuerte protagonismo. Sobre estos recae gran parte de la responsabilidad y compromiso de los cuidados, el control y supervisión del tratamiento, el seguimiento de la evolución de la enfermedad, etc., con el apoyo de los equipos y unidades de salud mental.

Es desde ese preciso instante que los poderes públicos y la administración del Estado dan paso a un modelo de intervención sociosanitario donde paciente, familia, profesional sanitario y comunidad entran en juego. En ellos descansa gran parte de la responsabilidad social y sanitaria hacia la enfermedad, quedando así instaurado un nuevo sentir e instalándose una nueva conciencia del deber cumplido. Habiendo pasado la administración pública a un segundo plano, el compromiso ético y moral adquirido por las familias de estos pacientes las obliga a desarrollar y poner en marcha estrategias de actuación para las que quizás no estén del todo preparadas. La falta de apoyos formales y de recursos sanitarios y sociales, agravados por la crisis económica en que nos encontramos sumidos, hacen de la familia un blanco fácil sobre el que apuntar a la hora de conciliar dichas deficiencias e irregularidades. En tanto que suplentes encargados de hacer frente a estos desajustes, nos encontramos con un moderado índice de familias donde la persona que ejerce los cuidados de larga duración o seguimiento de este perfil de pacientes (cuidadores informales) supera los 65 años, edad que limita el ejercicio de las funciones que le vienen encomendadas.

Es recomendado desde los poderes públicos y los sistemas sanitarios enfatizar en colectivos específicos que por su enfermedad invalidante o discapacitante, pueden verse inmersos en situaciones de alto riesgo social y sanitario e inermes por su propio 
estado de salud y el continuo cambio social (Jiménez, 2015: 13-18). El apoyo a las familias y sus cuidadores ha de estar en primera línea de debate y ser priorizado para la continuidad de unos cuidados formales e informales sostenibles y de calidad en estos pacientes (Silberstein, 1998: 25-30). Contribuyendo con el respiro y desahogo de dichos familiares cuidadores, se podrá evitar el debilitamiento, cansancio, agotamiento y renuncia del cuidado prestado (Morales et al., 2000; Delicado, 2003). Es por ello que se hace necesario analizar el sistema de protección social de este grupo de pacientes y sus familiares/cuidadores, conforme queda establecido en la Ley de Promoción de la Autonomía Personal y Atención a las personas en situación de dependencia, que permita desarrollar líneas de actuación que garanticen la continua cobertura de servicios y prestaciones, y aseguren la estabilidad clínica.

\section{OBJETIVOS DEL ESTUDIO}

Es motivo de nuestra investigación determinar, tanto la proporción de pacientes diagnosticados de TMG por el Equipo de Salud Mental Comunitaria (ESMC) de Palma del Río respecto del censo general de pacientes ratificados en Comisión del TMG, como describir la situación de dependencia en la que se encuentran dichos pacientes de acuerdo con la Ley 39/2006, de 14 de diciembre, de Promoción de la Autonomía Personal y Atención a las personas en situación de dependencia. Asimismo, nos interesa conocer el nivel de apoyo (formal e informal) con el que cuentan dichos pacientes y sus familiares por el estado de vulnerabilidad al que pueden verse sometidos dadas las circunstancias actuales del sistema de protección social y sanitario. Finalmente, y no menos importante, intentaremos delimitar la proporción de cuidadores o personas de referencia mayores de 65 años al cuidado o supervisión de pacientes psiquiátricos, por las limitaciones y crisis psicóticas a las que han de hacer frente, y el desgaste físico y psíquico (emocional) al que han de verse sometidos inexcusablemente.

\section{DISEÑO}

Dicha investigación pone su acento en los estudios de tipo observacional descriptivo transversal y cohorte cuantitativa.

\section{Emplazamiento}

Centramos nuestra atención en el grupo de pacientes con TMG diagnosticado, ratificados en la Comisión del TMG, adscritos al ESMC del municipio de Palma del Río, situado este en un enclave rural de la provincia de Córdoba (Valle Medio del Guadalquivir). 


\section{Material y método}

Para nuestro estudio hacemos uso del universo muestral de pacientes con trastorno mental grave diagnosticado, ratificados conforme a los procedimientos internos establecidos desde la dirección de la Unidad de Gestión Clínica (UGC) de Salud Mental del Hospital Universitario Reina Sofía (HURS) de la provincia de Córdoba, censados con fecha de 1 de enero de 2014 a 31 de diciembre de 2014 (tomamos como criterio de exclusión haber fallecido dentro del periodo señalado). Se recogen variables de tipo sociodemográfico (sexo, edad, estado civil, nacionalidad) y sanitarias (diagnóstico y persona de referencia). De igual manera, se recogen variables sociodemográficas de los cuidadores de estos pacientes, que determinarán de forma indirecta la capacidad y habilidad para la contención. Para el análisis descriptivo de los datos se hace uso del programa informático SSPS versión Statistics 22.0.

\section{RESULTADOS}

En nuestro estudio se incluyen 75 individuos (pacientes) adscritos al ESMC de Palma del Río, lo que representa un 10,8 \% del total de individuos censados (693 pacientes) conforme a la Comisión del TMG. La edad media es de 44,7 años (rango 24-70; Dt: $9,2)$. El $77,3 \%$ de individuos son varones y el $100 \%$ de nacionalidad española. El $72 \%$ está soltero/a y el 66,7 \% reside en el domicilio familiar (ver Figuras 1 y 2).

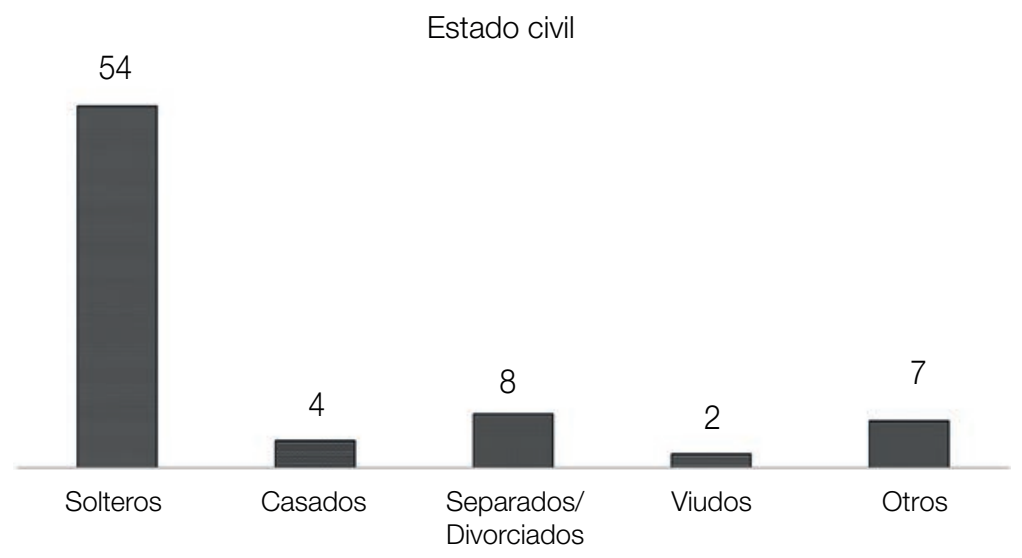

Figura 1: Estado civil del paciente con TMG en Palma del Río.

Fuente: Elaboración propia. Datos obtenidos del censo TMG y de Diraya Especializada. Diciembre, 2014. 


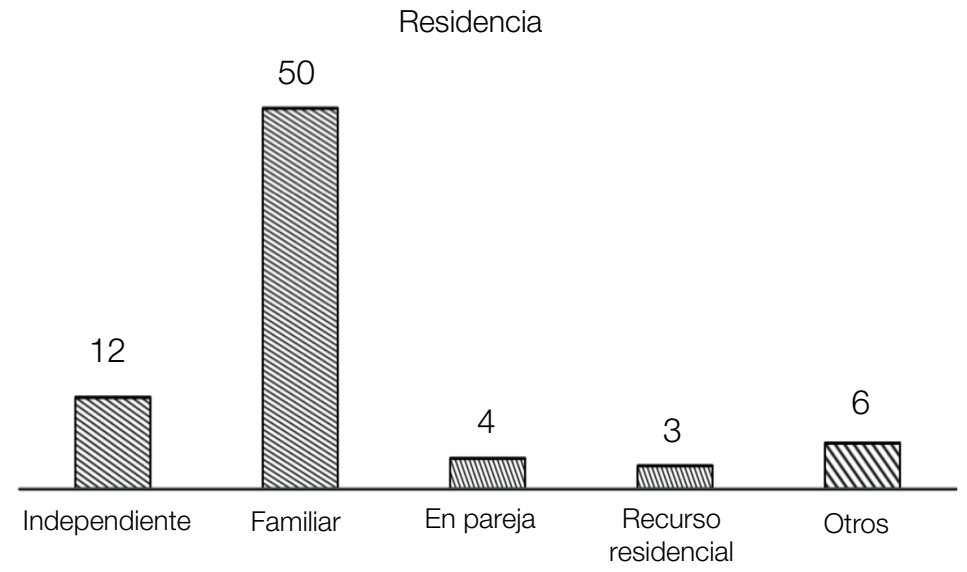

Figura 2: Situación residencia del paciente con TMG.

Fuente: Elaboración propia. Datos obtenidos del censo TMG y de Diraya Especializada; Diciembre, 2014.

Respecto de la patología predominante, el 68 \% lo constituyen aquellos pacientes incluidos en el bloque de las esquizofrenias (F.20 y sus variantes, según la CIE-10), seguido con un $16 \%$ por aquellos pacientes con trastorno esquizoafectivo y con un 10,6 \% por los pacientes diagnosticados de trastorno bipolar (ver Figura 3).

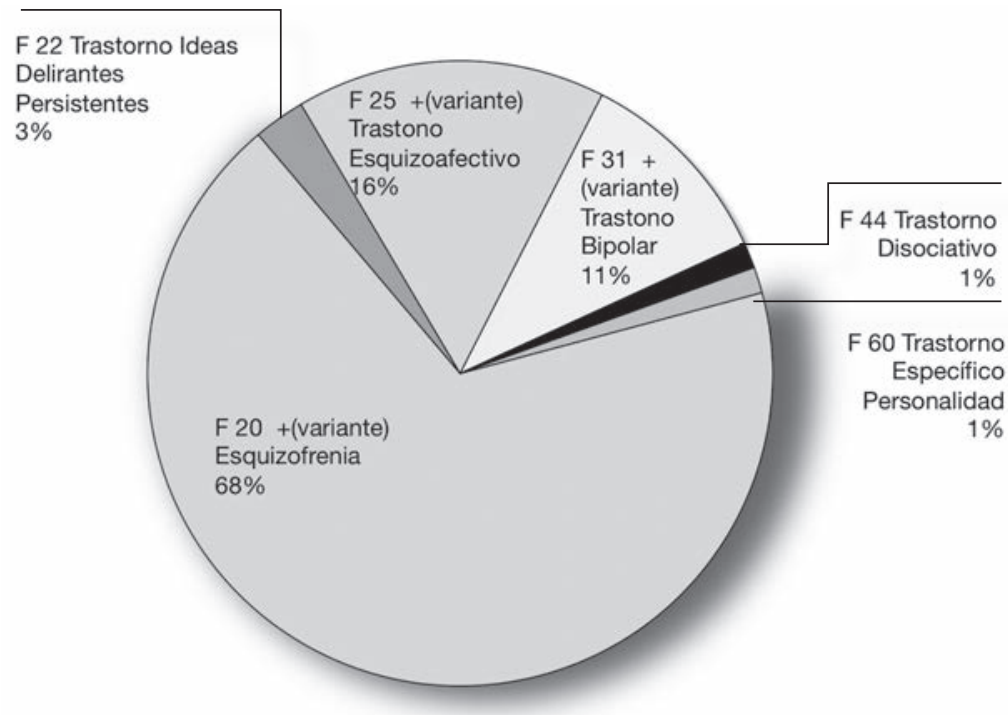

Figura 3: Especificidades por diagnósticos de los pacientes con TMG en el ESMC de Palma del Río según la CIE-10.

Fuente: Elaboración propia. Datos obtenidos del censo TMG y de Diraya Especializada; Diciembre, 2014. 
En cuanto a la situación de dependencia de dichos individuos (pacientes), el 49,3\% está valorado conforme a la Ley 39/2006, de 14 de diciembre, de Promoción de la Autonomía Personal y Atención a las personas en situación de dependencia, y el 2,7 \% la tiene solicitada (ver Figura 4).

Situación de dependencia

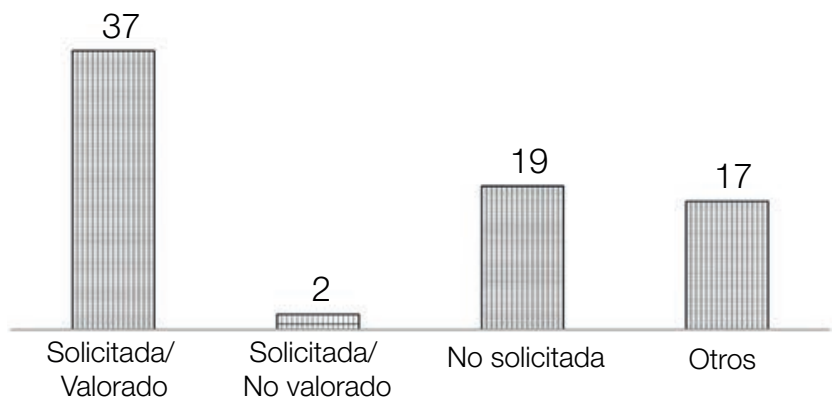

Figura 4: Descripción de la situación de dependencia de los pacientes con TMG en el ESMC de Palma del Río.

Fuente: Elaboración propia. Datos obtenidos del censo TMG y de Diraya Especializada; Diciembre, 2014.

El 20,3\% de estos individuos tiene prestación concedida, representando el Servicio de Ayuda a Domicilio (SAD) y la ayuda económica el 17,4 \%, seguido del recurso residencial con el 2,9 \% (ver Figura 5).

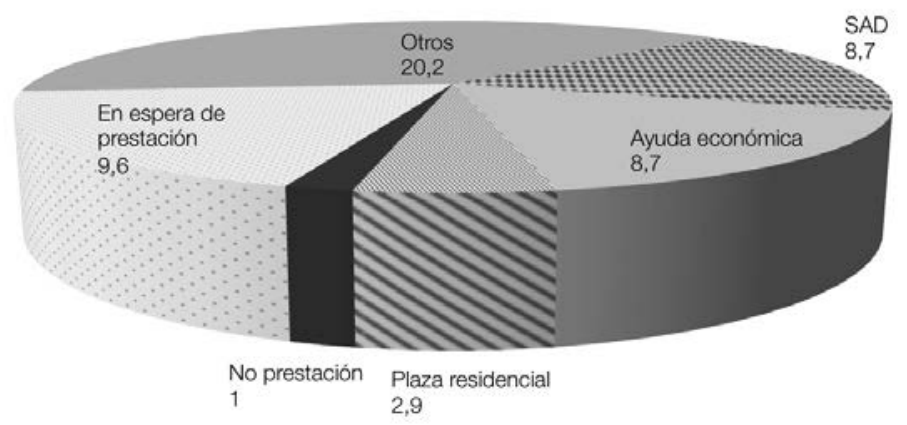

Figura 5: Distribución de prestaciones vía dependencia de los pacientes con TMG en el ESMC de Palma del Río.

Fuente: Elaboración propia. Datos obtenidos del censo TMG y de Diraya Especializada; Diciembre, 2014. 
Finalmente, respecto de los apoyos y cuidados con que cuentan estos pacientes cabe decir que, el $51 \%$ de los pacientes cuenta con una persona de referencia, sea esta un familiar directo o cónyuge, frente al 17,3 \% que no requiere de esta figura o de persona cuidadora (datos obtenidos de la Historia Clínica y Plan Individual de Tratamiento del paciente). El 32,7 \% de las personas cuidadoras y personal de referencia son mujeres y el 32,7 \% es mayor de 65 años.

\section{CONCLUSIONES}

El Trastorno Mental Grave y las patologías psiquiátricas en su conjunto han de comportar prioridad asistencial respecto de otras patologías menos invalidantes. Son los propios pacientes y sus familiares quienes sufren de primera mano los efectos de una enfermedad crónica marcada por desajustes y descompensación clínica, así como por la falta de respuesta institucional en determinados casos donde las medidas de protección social tienden a la remisión y el desvanecimiento. Recae sobre los familiares de referencia de estos pacientes (cuidadores informales) la asunción de las limitaciones y deficiencias de un sistema de protección social marcado por la austeridad y la recesión económica. La propia cronicidad de la enfermedad, la fluctuación evolutiva de esta y los cuidados (apoyo) de larga duración, entre otros, provocan cansancio y debilitamiento en los familiares de referencia o cuidadores quienes, en ocasiones, cuentan con una edad superior a los 65 años. Dichos factores, unidos al enlentecimiento y merma en la concesión de prestaciones propuestas por la Delegación de Igualdad, Salud y Políticas Sociales de la Junta de Andalucía, y más concretamente por la Ley 39/2006, de 14 de diciembre, de Promoción de la Autonomía Personal y Atención a las personas en situación de dependencia hacen que, tanto el paciente y la familia como los propios equipos de salud mental comunitaria, se vean afectados y desprovistos de respuestas sociosanitarias adecuadas a las exigencias de cada caso.

Estas circunstancias pueden dar como resultado una consunción física y emocional por parte de los cuidadores quienes, en un acto involuntario de salvaguardar la propia integridad psíquica y su salud mental, por el estrés que pueda generar el cuidado de un paciente con trastorno mental grave, obstaculicen el desarrollo y favorecimiento de los intereses y necesidades de estos. El paciente con TMG (consciente de su enfermedad y adherente al tratamiento) suele vivir ajustado y con una adecuada calidad de vida, pero la cronicidad y los periodos variables de agitación clínica llevan aparejados inestabilidad emocional y miedo a la recaída. Este hecho hace que se plantee como ineludible la constante supervisión y seguimiento de estos pacientes que, para el 66,6 \% de los casos analizados, se ven obligados a vivir en el domicilio familiar. Se hace necesaria desde los poderes públicos, los sistemas sanitarios y la sociedad en general una apuesta segura y mesurable, la cual brinde la posibilidad a pacientes y familiares de adquirir apoyos y ayuda en lo que respecta a la asistencia, el cuidado y la continui- 
dad del mismo, y que garantice la integración y normalización clínica. Es aquí donde la figura del trabajador social en los ESMC es de suma importancia pues su labor y cometido, su profesionalidad y entrenamiento, etc., dotan de habilidad y criterio a los citados equipos de salud mental, y son el complemento a la medicina asistencialista y el diagnóstico clínico.

\section{BIBLIOGRAFÍA}

Delicado, M.V. (2003). Familia y Cuidados de Salud: Calidad de Vida y repercusiones socio-familiares de la dependencia [tesis doctoral]. Alicante.

Gisbert, C., Arias, P., Camps, C., Cifre. A., Chicharro., F., Fernández J., et al. (2002). Rehabilitación psicosocial del trastorno mental severo: situación actual y recomendaciones. Madrid: Asociación Española de Neuropsiquiatría. Cuadernos Técnicos, $n^{\circ} .6$.

IMSERSO. (2007). Modelo de atención a las personas con enfermedad mental grave. Col. Documentos. Serie Documentos Técnicos.

Jiménez, J.M. (2015). El alta hospitalaria de pacientes con trastorno mental grave ingresados en la unidad hospitalaria de salud mental en la provincia de Córdoba: el papel del trabajador social. TS Difusión, (108), 13-18.

Laviana, M. (2006). La atención a las personas con esquizofrenia y otros trastornos mentales graves desde los servicios públicos: una atención integral e integrada en un modelo comunitario. Apuntes de Psicología, (24), 345-373.

Ley 39/2006, de 14 de diciembre, de Promoción de la Autonomía Personal y Atención a las personas en situación de dependencia. BOE n. ${ }^{2} 299$, de 15 de diciembre de 2006.

Morales, J.A., Muñoz, T., Bravo, F., Iniesta, C., Montero, F.A., y Olmos, M.C. (2000). Problemas de salud de los cuidadores de enfermos incapacitados. Centro de Salud, (8), 714-718.

OMS (2003). CIE-10 Clasificación estadística internacional de enfermedades y problemas relacionados con la salud. $10^{\mathrm{a}} \mathrm{Ed}$. rev. Washington.

Schinnar A.P., Rothbard, A.B., Kanter, R., y Jung, Y.S. (1990). An empirical literature review of definitions of severe and persistent mentalillness. Revista Americana de Psiquiatría, 147, (12), 1602-8.

Silberstein, D. (1998). La familia, un paciente olvidado. Rev Rol Enf, (243), 25-30. 
